\title{
Optical probing of the temperature and pressure transients at a liquid/solid interface due to pulsed laser-induced vaporization
}

\author{
Hee K. Park ${ }^{1}$, Costas P. Grigoropoulos ${ }^{2}$, Chie C. Poon ${ }^{2}$, Andrew C. Tam², Oguz Yavas ${ }^{3}$, and Paul Leiderer ${ }^{3}$
}

${ }^{1}$ Department of Mechanical Engineering, University of Califomia

Berkeley, California 94720

${ }^{2}$ IBM Almaden Research Center, 650 Harry Road

San Jose, California 95120-6099

${ }^{3}$ Department of Physics, University of Konstanz

D-78434 Konstanz, Germany

\begin{abstract}
The transient temperature and pressure field development in the excimer laser-induced vaporization of liquids in contact with a solid surface is studied. A thin silicon film, which has temperature-dependent optical properties, is embedded between an absorbing chromium film and a transparent fused quartz substrate. Static reflectivity measurement is performed to determine the thin film optical properties at elevated temperatures. The transient backward reflectance responses from the silicon layer are compared with heat transfer modeling results. The backward reflectance probe is not affected by the creation of bubbles and is successfully employed for the first time to measure non-intrusively the temperature development during the rapid vaporization process. The optical reflectance probes are applied from the front-side and back-side of the sample simultaneously to monitor the dynamic bubble nucleation behavior and transient temperature development, respectively, at various ambient pressures using a high-pressure cell. The investigation on the effect of ambient pressure on the bubble nucleation threshold combined with the surface temperature measurement determines the thermodynamic state of the superheated metastable liquid at the interface and subsequently the explosion pressure.
\end{abstract}

Keywords: laser-induced vaporization, heterogeneous nucleation, explosive vaporization, photothermal diagnostic method

\section{INTRODUCTION}

Laser beam interaction with materials in liquid provides a very unique laser processing environment ${ }^{1-3}$. The explosive vaporization of liquid films on the opaque solid surfaces heated by short-pulsed laser irradiation is utilized in laser-assisted cleaning of microcontaminants ${ }^{4}$ and the liquid-film-enhanced laser-hardening of materials ${ }^{5}$. Shortpulsed laser-induced nucleation and cavitation in a tissue or an organ produces a sharp incision with minimal injury which enables the surgical operation in great accuracy. The therapeutic use of a laser instead of a surgical knife is already practiced clinically to a large extent, from the intraocular surgery in ophtbalmology ${ }^{6}$ to laser lithotripsy for the fragmentation of bilinary and urinary calculi ${ }^{7,8}$ and laser angioplasty for the treatment of in cardiovascular disease $^{9}$.

The physical understanding of superheated pure or mixed liquids, such as limit of superheat and critical phenomena, has been sought for a better control of such technical applications. The science of metastability of liquid has been established in the near-steady-state regime. There have been efforts to understand the thernodynamics of the rapid vaporization of liquids on an absorbing solid surface heated by nanosecond-pulsed laser irradiation. The transient development of the bubble nucleation process and the onset of phase change are monitored by simultaneous application of optical reflectance and scattering probes ${ }^{10}$. The reflectance and scattering signals show the distinct transients of nucleation and growth of bubbles at the liquid-solid interface when the excimer laser fluence exceeds a certain liquid-dependent threshold ${ }^{10}$. The numerical heat conduction calculation also shows that the solid surface achieves temperatures of tens of degrees of superheat ${ }^{11}$. However, no real-time measurement of the surface temperature development in the course of the laser-induced vaporization process has been 
made, although the sufface temperature (i.e., the wall superheat) is the most important parameter in the heterogeneous nucleation phenomenon ${ }^{12,13}$. The thermodynamic considerations on the problem including the degree of superheat required for a liquid-vapor transition in nanosecond time scale have never been addressed. Disturbances by bubbles near the liquid-solid interface, the relatively low teroperature change, and the insensitivity of electric and optical properties of metals on temperature have prohibited the precise detection of surface temperature during the laser-induced vaporization. Recently, it has been proven that the transient optical transmission or reflectivity probe is a fast, reliable method with nanosecond resolution to monitor the temperature field development during the processing of semiconductor films by short-pulsed laser ${ }^{14-18}$. In this work, the optical reflectance probe is applied from the backside to the embedded thin semiconductor film. The backward reflectance probe is not affected by the creation of bubbles in the liquid and is successfully employed to measure the temperature field development during the rapid vaporization process.

The precise assessment of the value of pressure produced by the growing and collapsing bubbles is a critical step in designing the technical applications. The generation of strong pressure waves in liquid pools under intense short pulsed-laser irradiation has been investigated by various methods, for example, using hydrophone or piezoelectric transducers ${ }^{19-21}$, bigh-speed photography ${ }^{22}$, interferometry ${ }^{23}$, and optical probing ${ }^{24,25}$. However, most of optical (photographic) methods rely on the shock wave velocity measurements and the shock wave velocitypressure relations for the quantification of pressure ${ }^{26}$. In a lower energy regime where velocity is not supersonic, such a calibration is not applicable. The hydrophone or piezoelectric transducer probe also has a limit in general usage due to the transducer ringing and the lack of bandwidth. In this work, a systematic study is performed on the pressure generation by monitoring the bubble growth kinetics combined with the photothermal temperature measurement at several ambient pressures in a pressure cell. Hence, the thermodynamics state is determined for the rapid vaporization of liquids. Consequently, the precise evaluation on the pressure level and the metastability behavior of liquid is obtained.

\section{EXPERIMENTAL ARRANGEMENT AND PROCEDURE}

The experimental setup is shown in Fig. 1. A liquid is filled in a massive high pressure cell (maximum pressure $\sim 100 \mathrm{~atm}$ ) made of stainless steel and quartz windows. The pressure cell is designed to minimize the en-

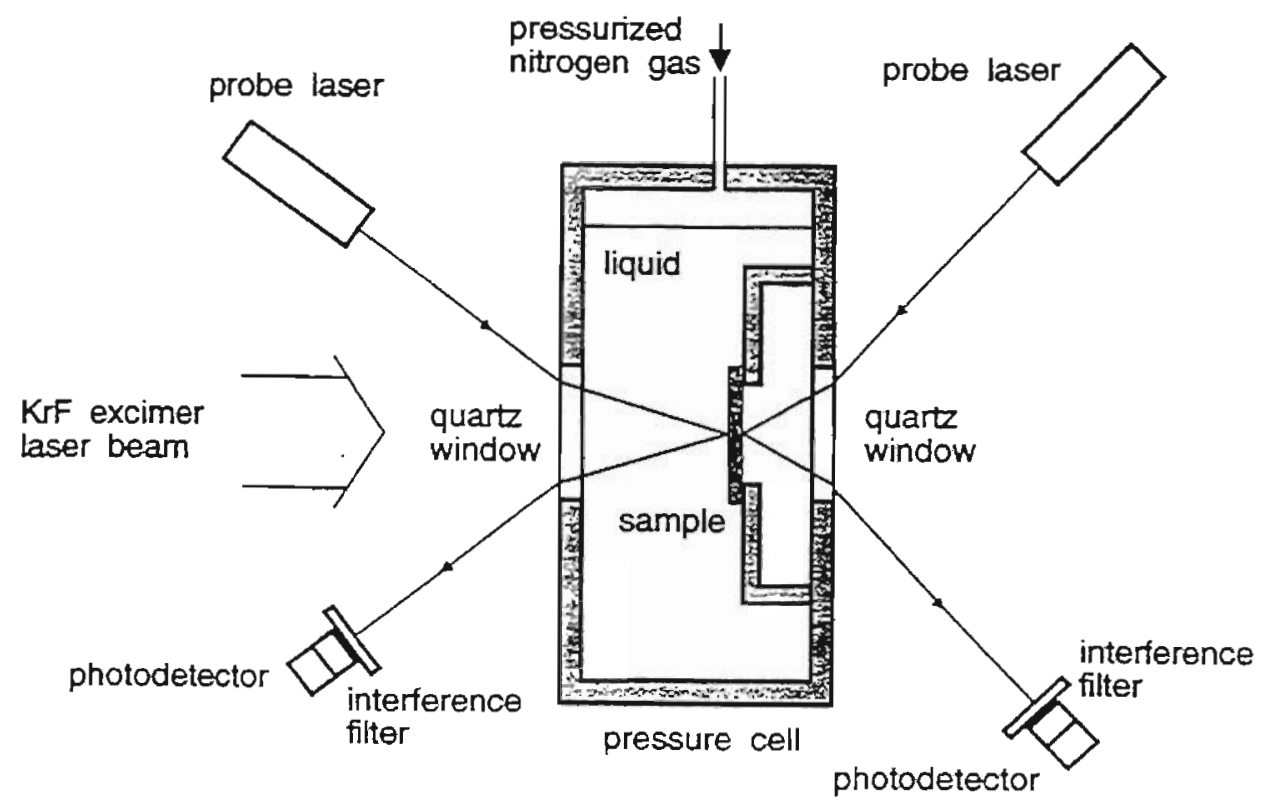

Fig. 1 Schematic of experimental setup. 
vironmental vibrations. The pressure of liquid is varied by applying compressed nitrogen into the pressure cell. The solid sample has 3 layers as shown in Fig. 2. The top layer is an absorbing film, the intermediate layer is an optical temperature sensor whose optical properties vary with temperature, and the bottom layer is a transparent substrate. In the temperature monitoring schezae of this work, the change of optical properties, i.e, the change of the temperature, is probed by the photothermal reflectance of the intermediate layer irradiated from the backside (substrate side). The advantage of the probe from the backside is the ability of avoiding the occurrence of events neat or on the laser irradiated surface such as bubble nucleation. In this study, chromium is selected as a top layer material, polycrystalline silicon (p-Si) as an optical sensor, and fused quartz as a substrate. The thickness of the cbromium layer is determined to be larger than the optical penetration depth at the excimer laser light wavelength, which is $9.8 \mathrm{~nm}$ for the bulk chromium. Therefore, the direct heating of the temperature sensor layer by the excimer laser will not occur. On the other hand, the thickness of the chromium layer is sufficiently smaller than the thermal penetration depth. The thermal penetration depth of chromium is $0.17 \mu \mathrm{m}$ at 1 ns. The optimum thickness of the temperature sensor layer has to be well designed for better temperature sensitivity. In order to maximize the temperature sensitivity, the thickness of the sensor film has to maximize $d \mathscr{R} / d T$, where $\mathscr{R}$ is the reflectivity of the sensor film. It is known that the change of reflectivity due to the change of refractive index is maximum when the optical thickness nd is similar to the wavelength. In this work, the sample with the 0.15 $\mu \mathrm{m}$-thick Cr film and $0.35 \mu \mathrm{m}$-thick p-Si film deposited on a $500 \mu$ m-thick fused quartz substrate is fabricated. Both sides of the surface are probed by two $\mathrm{CW}$ lasers. Since the $\mathrm{Cr}$ film has no intrinsic thermoreflectance effect, any change of the optical reflecance is ascribed to the formation of bubbles on the surface. On the other hand, due to the absence of bubbles in the backside, any change on the reflecance from the p-Si layer is only caused by the temperature change. Hence, the temperature and the bubble nucleation behavior can be obtained separately at the same time. The $\mathrm{KrF}$ excimer laser beam ( $\lambda=248 \mathrm{~nm}$, pulse width $=16 \mathrm{~ns}$ FWHM) is irradiated over a large area $\left(1 \times 1 \mathrm{~cm}^{2}\right)$ and absorbed by the $\mathrm{Cr}$ layer. The probe $\mathrm{CW}$ lasers are an $\mathrm{Ar}^{+}(\lambda=488 \mathrm{~nm})$ for the forward reflectance, and a HeNe laser $(\lambda=632.8 \mathrm{~nm})$ for the backward reflectance. It is noted that the incident angle for the forward reflectance is so small $\left(<10^{\circ}\right)$ that the photoacoustic deflection of the beam can be neglected.

\section{STATIC TEMPERATURE CALIBRATION}

First the photothermal reflectivity from the back-side of the sample is calibrated by the static experiment. The sample is mounted on an aluminum block of which temperature is controlled within $\pm 0.1^{\circ} \mathrm{C}$. The measured static reflectivity are fitted to obtain the optical properties of quartz substrate and p-Si film:

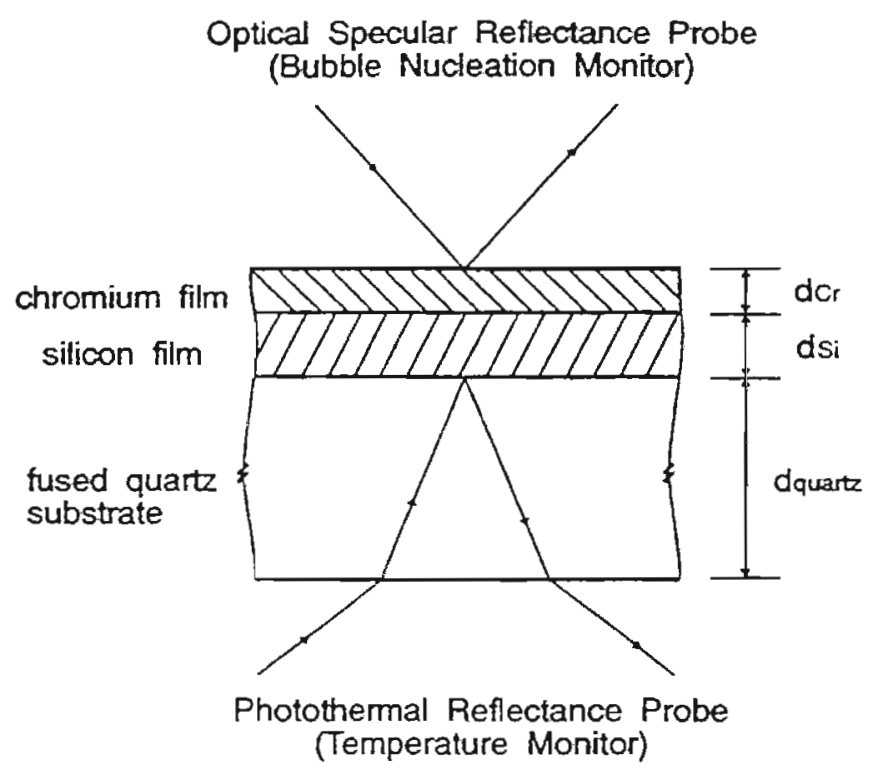

Fig. 2 Schematic of sample structure and optical detection scheme. 


$$
\begin{aligned}
& n_{\text {quart }}=1.457+9.64 \times 10^{-6} \times(T-20)+6.1 \times 10^{-9} \times(T-20)^{2} \\
& n_{p-S_{i}}=4.01+3.31 \times 10^{-4} \times(T-20) \\
& k_{p-S_{i}}=0.0355 \times \exp \left(\frac{T}{590}\right)
\end{aligned}
$$

where the temperature $T$ is in degree Celcius. It is noted that the obtained optical properties are close to the literature values ${ }^{27}$. The accuracy of the fitting is better than $1 \%$. The optical property change of the fused quartz does not affect the transient temperature measurement in nanosecond time scale because of the poor thermal conductivity of quartz. The temperature profile in the sample is modelled by the one-dimensional heat diffusion equation. Detailed procedure on the temperature modeling appear elsewhere ${ }^{17}$. In the calculation of the theoretical reflectivity response, a stratified-layer approach is utilized to take an account of the steep temperature gradient across the sample in the nanosecond time scale ${ }^{17}$. In this approach, the sample is assumed to be comprised of multiple layers with the calculated temperature and corresponding optical properties determined by the static calibration. The reflectivity is calculated by the thin-film matrix method ${ }^{28}$.

\section{TRANSIENT TEMPERATURE MEASUREMENT}

Figure 3 shows the results of the transient temperature measurement. The excimer laser irradiates the water/chromium interface of the sample at the fluence of $42.2 \mathrm{~mJ} / \mathrm{cm}^{2}$ at atmospheric pressure. The top panel shows a slight change in the forward reflectance, indicating the water-chromium interface is right at the threshold for nucleation. The signal shown in the figure is an average of 5 shots of laser pulses so as to minimize the noise level. The middle panel shows the experimental photothermal reflectance curve (solid line) and the theoretical

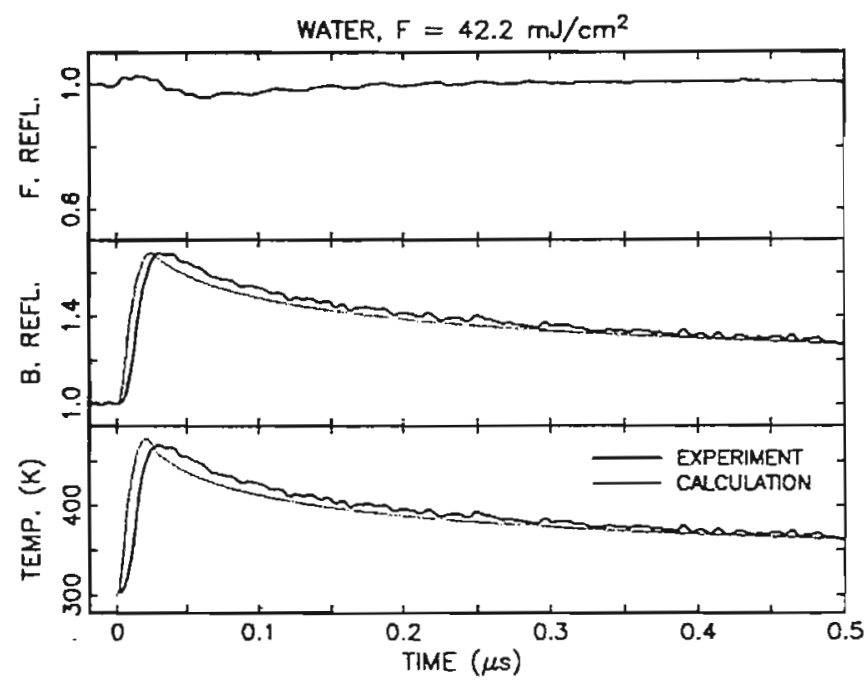

Fig. 3 The experimentally obtained reflectance curves (solid lines) are shown in the top panel (forward reflectance) and middle panel (backward photothermal reflectance) for water. The dotted lines are calculated transient reflectivity response (middle panel) and surface temperature (bottom panel). The converted surface temperature from the measured reflectance is shown for comparison. The excimer laser fluence is $42.2 \mathrm{~mJ} / \mathrm{cm}^{2}$.

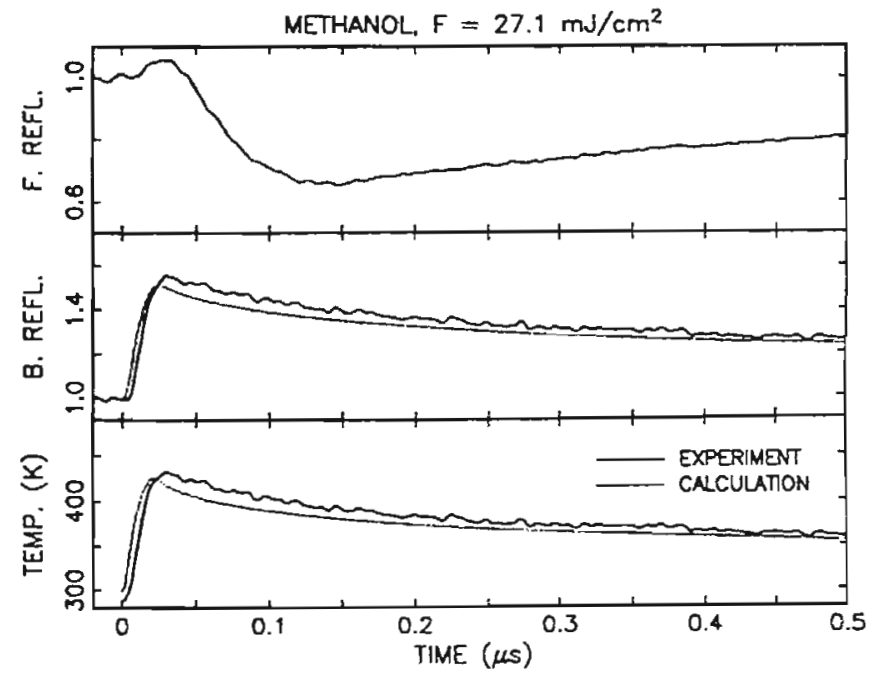

Fig. 4 The experimentally obtained reflectance curves (solid lines) are shown in the top panel (forward reflectance) and middle panel (backward photothermal reflectance) for methanol. The dotted lines are calculated transient reflectivity response (middle panel) and surface temperature (bottom panel). The converted surface temperature from the measured reflectance is shown for comparison. The excimer laser fluence is $27.1 \mathrm{~mJ} / \mathrm{cm}^{2}$. 
photothermal reflectance response (dotted line). The measured (solid line) and calculated (dotted line) evolutions of surface temperature are compared in the bottom panel. The measured surface temperature trace is converted from the obtained reflectance signal. The measured maximum surface temperature value is the same as the calculated, $476 \mathrm{~K}$, which is $103 \mathrm{~K}$ above the boiling temperature.

The transient temperature measurement for the methanol/chromium interface of the sample at the fluence of $27.1 \mathrm{~mJ} / \mathrm{cm}^{2}$ at atmospheric pressure is shown in Fig. 4. A drop in the forward reflectance is seen in the top panel of the figure. Hence bubble nucleation takes place at this fluence level. By repeated measurements, the threshold for nucleation is determined to be $23.5 \mathrm{~mJ} / \mathrm{cm}^{2}$. The temperature measurement on methanol results in the maximum surface temperature of $422 \mathrm{~K}$ and the calculation yields $409 \mathrm{~K}$. Consequently the degree of superheat required for the nucleation in the nanosecond heating is approximately $80 \mathrm{~K}$ (boiling point $=337.9 \mathrm{~K}$ ).

The measured peak surface temperature at atmospheric pressure is plotted as a function of the excimer laser fluence in Fig. 5 for water. The temperature is approximately linear with the fluence. The discrepancy between the measurement and calculation arises from the possible departure of thermal properties of thin films from bulk values and the existence of contact resistance between layers. Nevertheless, it can be stated that the technique used in this work can measure the surface temperature with accuracy of $30^{\circ} \mathrm{C}$. The different slope of the temperature rise versus fluence between experiment and calculation is due to the increasing departure of properties from bulk values at higher temperatures. It is also in part due to the assumption in the computation that no phase change is taken into account. This is a fair assumption, however, because a large fraction of heat is transferred to solid and only a small fraction of the absorbed heat is transferred to the liquid. Therefore, the temperature field is not strongly affected by the occurrence of vaporization and the interface temperature can be well estimated by the simple conduction equation. It is however noted that the trend of lower measured temperature compared with the calculated values at higher laser fluence suggests the increasing amount of vaporization at higher temperatures. It is also observed in methanol as illustrated in Fig. 6. The trend of lower measured temperature than calculated temperature is more pronounced in methanol. Generally, experimental data of methanol are not as good as those of water. This is attributed to the lower temperature rise of methanol, i.e., worse signal-to-noise ratio, and higher volatility. Error bars are indicated in Fig. 6. The limit of superheat of methanol is $477 \mathrm{~K}^{29}$. Surface temperature exceeding the limit of superheat is also measured (see Fig. 6). At this condition, it is expected that the liquid adjacent to the surface would completely evaporate, forming a vapor film. This hypothesis is indeed confirmed experimentally. Figure 7 shows the forward reflectance transients for increasing laser fluences. When the surface temperature is below the limit of superheat at $30.3 \mathrm{~mJ} / \mathrm{cm}^{2}$, the forward reflectance curve shows typical bubble growth and collapse signatures (see Fig. 4). At higher fluences, the experimental reflectance curve shows the characteristics of a vapor film. First, the

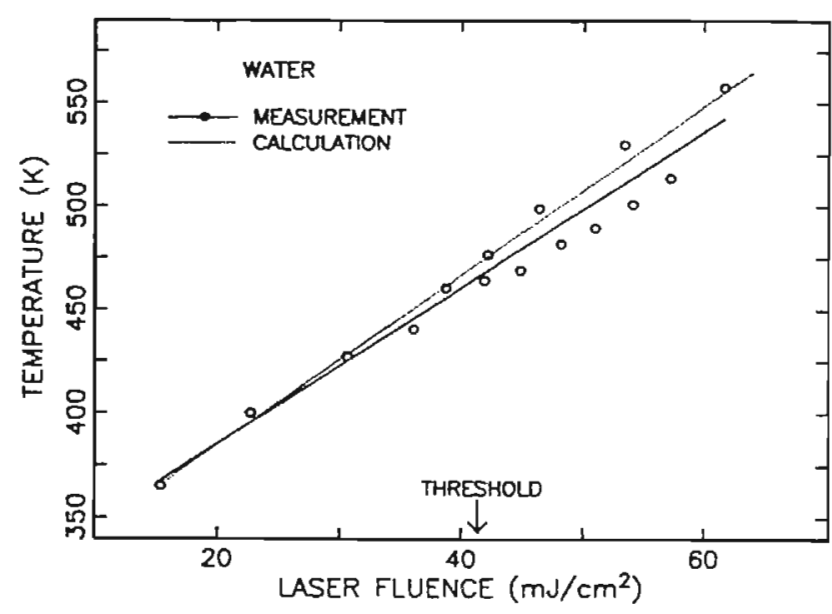

Fig. 5 The measured surface temperature is compared with the calculated surface temperature as a function of laser fluence for water. The nucleation threshold is indicated by arrow. Solid line is a linear fitting of experimental data.

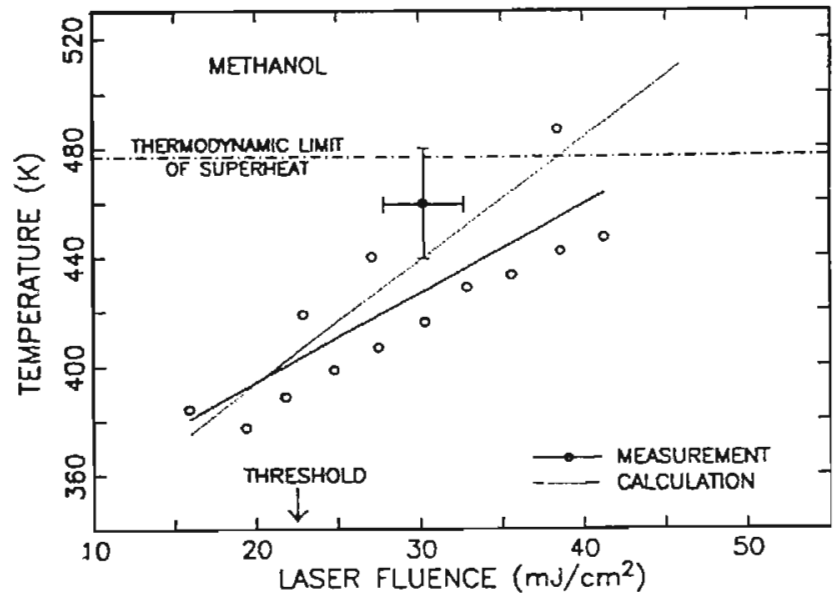

Fig. 6 The measured surface temperature is compared with the calculated surface temperature as a function of laser fluence for methanol. The nucleation threshold is indicated by arrow. Solid line is a linear fitting of experimental data. 


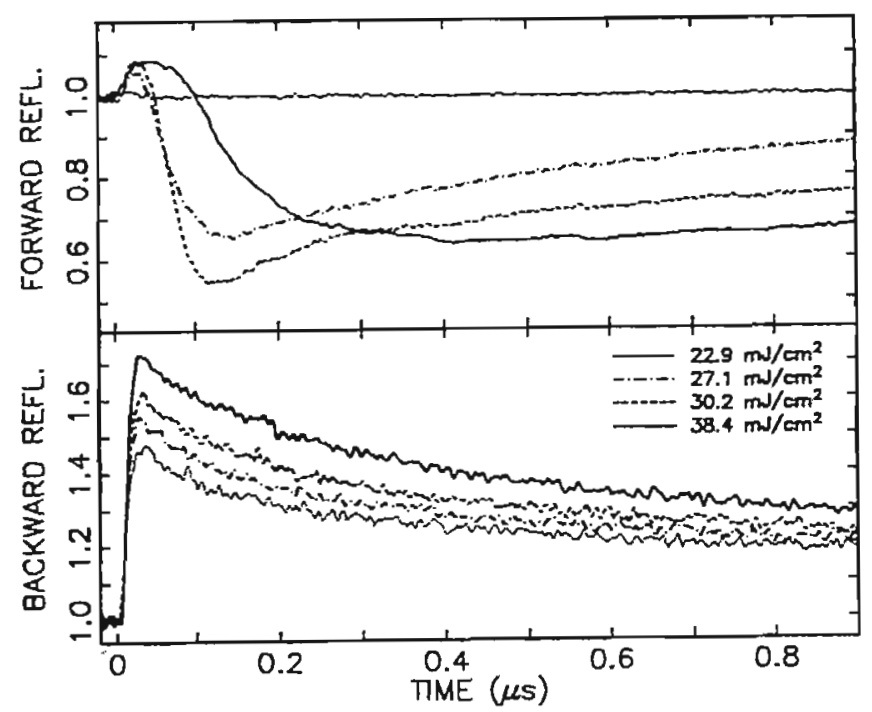

Fig. 7 The comparison of transient reflectance traces at various laser fluences for methanol.

amount of the reflectance drop is smaller, i.e., the scattering loss is smaller. It is known that a smooth vapor film will scatter less light than a cloud of bubbles. Secondly, the evolution of signal is much slower. Once a vapor film forms, the creation of bubbles (drop of the reflectance signal) is delayed until the vapor film collapses and breaks up due to cooling. Since the limit of superheat of water is very high $(596 \mathrm{~K})^{29}$, such an effect has not been observed for water.

\section{BUBBLE GROWTH DYNAMUCS IN HIGH PRESSURE}

The change of the specular reflectance in a high-pressure cell is monitored for the measurement of pressure. The transient signatures of the forward specular reflectance at various pressure levels at the fixed excimer laser

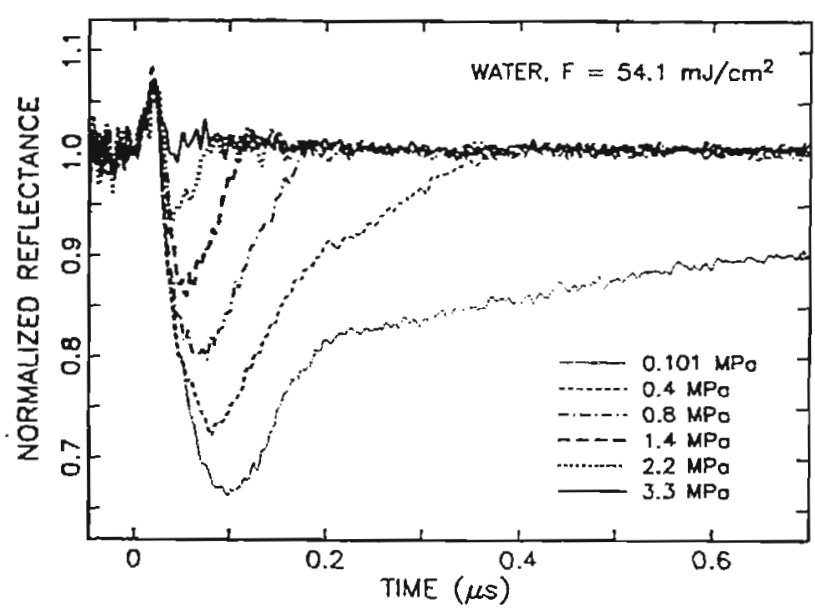

Fig. 8 The set of specular reflectance signals at various liquid pressures for water. The excimer laser fluence is $54.1 \mathrm{~mJ} / \mathrm{cm}^{2}$. The probe beam is $\mathrm{s}-$ polarized $\mathrm{Ar}^{-}$laser $(\lambda=488 \mathrm{~nm})$ at an incident angie of $10.5^{\circ}$.

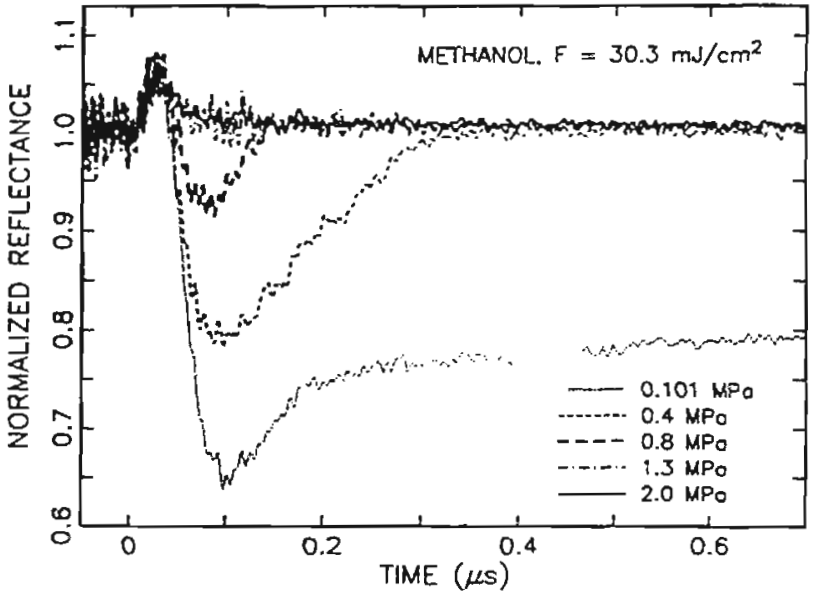

Fig. 9 The set of specular reflectance signals at various iiquid pressures for methanol. The excimer laser fluence is $30.3 \mathrm{~mJ} / \mathrm{cm}^{2}$. The probe beam is $\mathrm{s}-$ polarized $\mathrm{Ar}^{+}$laser $(\lambda=488 \mathrm{~nm})$ at an incident angle of $10.5^{\circ}$. 
Table 1 The measured peak surface temperature and cut-off pressure of the chromium sample. The saturation vapor pressure values at the measured peak temperature are provided for reference.

\begin{tabular}{|c|c|c|c|c|}
\hline \multirow{2}{*}{ Liquid } & $\begin{array}{c}\text { Laser fluence } \\
\left(\mathrm{mJ} / \mathrm{cm}^{2}\right)\end{array}$ & $\begin{array}{c}\text { Peak surface temper- } \\
\text { ature }(\mathrm{K})\end{array}$ & $\begin{array}{c}\text { Cut-off pressure } \\
(\mathrm{MPa})\end{array}$ & Vapor pressure (MPa) \\
\hline \multirow{3}{*}{ Water } & 44.9 & 468.8 & 1.7 & 1.42 \\
\cline { 2 - 5 } & 48.2 & 481.4 & 2.9 & 1.84 \\
\cline { 2 - 5 } & 54.1 & 500.8 & 3.5 & 2.68 \\
\hline \multirow{2}{*}{ Methanol } & 24.8 & 398.5 & 0.8 & 0.75 \\
\cline { 2 - 5 } & 30.3 & 416.0 & 1.6 & 1.17 \\
\hline
\end{tabular}

fluence are shown in Fig. 8 for water and Fig. 9 for methanol. The initial increase of the reflectance is due to the interference of the thin layer of small bubbles ${ }^{10}$. The nucleation of bubbles at the stage of initial rise of signal where the bubble size is $\ll \lambda / 2 \pi n$ (Rayleigh scattering regime) ${ }^{10}$ is nearly insensitive to the increase of ambient pressure. This finding indicates that the transition temperature of liquid to vapor is not sensitive to the pressure. It agrees with the fact that the limit of superheat, i.e., the liquid spinodal, is only a very weak function of pressure. It is, however, clearly seen that the negative amplitude of the reflectance, i.e., the growth of large bubbles $(\gg \lambda / 2 \pi n$ : Mie scattering regione), decreases with the ambient pressure. At $p_{t}=3.3 \mathrm{MPa}$ for water and at $p_{t}=1.3 \mathrm{MPa}$, the bubble growth is completely suppressed so that the negative phase of the specular reflectance is not observed. The negative drop of the reflectance is an indicator of the number and the size of bubbles causing Mie scattering $(r \gg \lambda / 2 \pi n)$ i0,11. Figures 10 and 11 display the magnitude of the negative drop of the specular reflectance as a function of liquid pressure for water and methanol, respectively. The zero reflectance drop indicates that no bubble much bigger than $\lambda / 2 \pi n$ is detectable. Therefore the "cut-off pressure" is defined as the minimum liquid pressure at which no bubble with size much bigger than $\lambda / 2 \pi n$ is generated, i.e., as the intercept of the reflectance drop curve on the abscissa. The measured cut-off pressure is summarized in Table 1 for water and methanol at various laser fluences. The measured surface temperature and corresponding saturation pressure are also listed. It is noted that the cut-off pressure is higher than the saturation pressure. Under thermal equilibrium, the condition for bubble formation is that the vapor pressure must be larger than the ambient liquid pressure. Therefore the difference between the cut-off

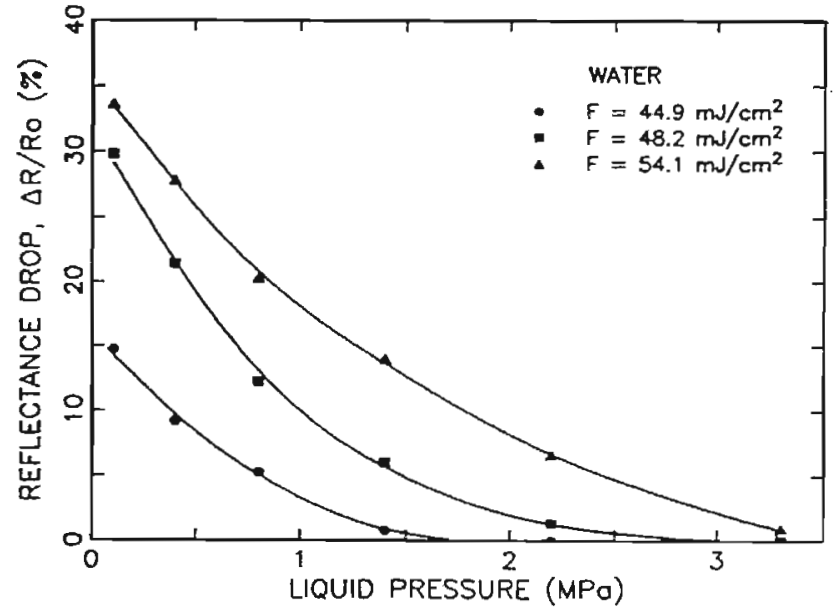

Fig. 10 The amplitude of the negative reflectance is plotted versus the liquid pressure for water at various excimer laser fluences. Curves are spline fits of experimental data (symbols).

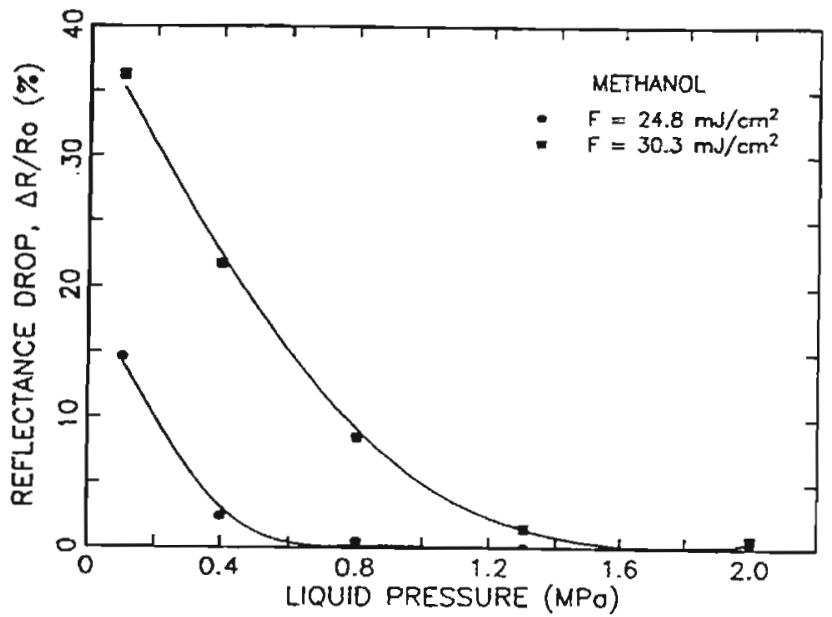

Fig. 11 The amplitude of the negative reflectance is plotted versus the liquid pressure for methanol at various excimer laser fluences. Curves are spline fits of experimental data (symbols). 
pressure and the saturation vapor pressure $p_{\text {sar }}\left(T_{l}\right)$ is an amount to be accounted for nonequilibrium vaporization. This can be understood as an excessive pressure force preventing further bubble growth or as a dynamic pressure generated by the exploding bubbles. The excessive pressure $p_{e x}$ is defined as the difference between the cut-off pressure and the saturation vapor pressure, i.e., $p_{c x}=p_{\text {cur }- \text { off }}-p_{s a r}\left(T_{l}\right)$. Hence $p_{c x}$ is a measure of the transient component of pressure generated upon vaporization and is of the order of $1 \mathrm{MPa}$ in Table 1 . It is noted in Table 1 that $p_{e x}$ increases with laser fluences. Therefore, the transient force is greater when the laser fluence is higher, i.e., the surface is hotter.

\section{CONCLUSION}

Transient optical techniques including photothermal reflectance and specular reflectance probes have been utilized together with a high-pressure cell to obtain quantitative temperature and pressure values during the laserinduced vaporization of liquids on a solid surface. Two time-resolved optical probes for bubble growth kinetics and temperature measurements are applied simultaneously in a high-pressure cell at elevated ambient pressures. The transient temperature measurement revealed that liquids can sustain about a hundred of degrees of superheat before the vaporization occurs. It has been found that bubble growth can be suppressed under tens of atmospheric pressure. It has been also estimated that pressure level generated by the explosive bubble growth is of the order of a few atmospheric pressure.

\section{ACKNOWLEDGEMENTS}

The authors thank D. Krajnovich and I. K. Pour of IBM Almaden Research Center for their valuable contributions to this work. Support to this work by the National Science Foundation under Grant CTS-9317708 is gratefully acknowledged.

\section{REFERENCES}

1. N. Morita, S. Ishida, Y. Fujimori, and K. Ishikawa, "Pulsed laser processing of ceramics in water," Appl. Phys. Lett., Vol. 52, pp. 1965-1966, 1986.

2. A. Polman, W. C. Sinke, F. W. Saris, M. J. Uttormark, and M. O. Thomson, "Quench rate enhancement in pulsed laser melting of Si by processing under water," Appl. Phys. Lett., Vol. 52, pp. 535-537, 1988.

3. R. J. von Gutfeld and R. T. Hodgson, R.T., "Laser enhanced etching in KOH," Appl. Phys. Lett., Vol. 40, pp. 352-354, 1982.

4. A. C. Tam, W. P. Leung, W. Zapka, and W. Ziemlich, "Laser-cleaning techniques for removal of surface particulates," J. Appl. Phys., Vol. 71, pp. 3515-3523, 1992.

5. B. P. Fairand and A. H. Clauer, "Effect of water and paint coatings on the magnitude of laser-generated shocks," Opt. Comm., Vol. 18, pp. 588-591, 1979.

6. A. Vogel, P. Schweiger, A. Frieser, M. N. Asiyo, and R. Birngber, "Intraocular Nd:YAG laser surgery: Lighttissue interaction, damage range, and reduction of collateral effects," IEEE J. Quant. Elect., Vol. 26, pp. 2240-2260, 1990.

7. P. Teng, N. S. Nishioka, R. R. Anderson, and T. F. Deutsch, "Optical studies of pulsed-laser fragmentation of bilinary calculi," Appl. Phys. B, Vol. 42, pp. 73-78, 1987.

8. K. Kink, G. Delacrétaz, and R. P. Salathé, "Fragmentation process induced by nanosecond laser pulse," Appl. Phys. Lett., Vol. 22, pp. 26442646, 1992.

9. J. M. Isner and R. H. Clarke, "The current status of lasers in the treatment of cardiovascular disease," IEEE J. Quant. Elect., Vol. 20, pp. 1406-1420, 1984. 
10. O. Yavas, P. Leiderer, H. K. Park, C. P. Grigoropoulos, C. C. Poon, W. P. Leung, N. Do, and A. C. Tam, "Optical reflectance and scattering studies of nucleation and growth of bubbles at a liquid-solid interface induced by pulsed laser heating," Phys. Rev. Lett., Vol. 70, No. 12, pp. 1830-1833, 1993.

11. O. Yavas, P. Leiderer, H. K. Park, C. P. Grigoropoulos, C. C. Poon, W. P. Leung, N. Do, and A. C. Tam, "Optical and acoustic study of nucleation and growth of bubbles at a liquid-solid interface induced by nanosecond-pulsed laser heating," Appl. Phys. A , Vol. 58, pp. 407-415, 1994.

12. V. P. Carey, Liquid-Vapor Phase-Change Phenomena, Hemisphere, Washington, 1992.

13. V. P. Skripov, Metastable Liquids, John Wiley \& Sons, New York, 1974.

14. G. E. Jellison Jr., D. H. Lowndes, D. N. Mashburn, and R. F. Wood, "Time-resolved reflectivity measurements of silicon and germanium using a pulsed excimer KrF laser heating beam," Phys. Rev. B, Vol. 34, No. 4, pp. 2407-2415, 1986.

15. L. A. Lompré, J. M. Liu, H. Kurz, and N. Bloembergen, N., "Time-resolved temperature measurement of picosecond laser irradiated silicon," Appl. Phys. Lett. Vol. 43, pp. 168-170, 1983.

16. D. H. Lowndes, "Time-resolved optical transmission and reflectivity of pulsed-ruby-laser irradiated crystalline silicon," Phys. Rev. Lett., Vol. 48, pp. 267-271, 1982.

17. H. K. Park, X. Xu, C. P. Grigoropoulos, N. Do, L. Klees, P. T. Leung, and A. C. Tam, "Transient optical transmission measurement in excimer-laser irradiation of amorphous silicon films," Trans. ASME J. Heat Transfer, Vol. 115, pp. 178-183, 1993.

18. X. Xu, C. P. Grigoropoulos, and R. E. Russo, "Transient temperature measurement during pulsed excimer laser heating of thin semiconductor films," Trans. ASME J. Heat Transfer, in press, 1994.

19. H. Schoeffman, H. Schmidt-Kloiber, and E. Reichel, E., "Time-resolved investigations of laser-induced shock waves in water by use of polyvinylidenefluoride hydrophones," J. Appl. Phys., Vol.63, pp.46-51, 1988.

20. W. P. Leung and A. C. Tam, Noncontact monitoring of laser ablation using a miniature piezoelectric probe to detect photoacoustic puises in air," Appl. Phys. Lett., Vol.60, pp. 23-25, 1992.

21. A. A. Oraevsky, S. L. Jacques, and F. K. Tittel, "Determination of tissue optical properties by piezoelectric detection of laser-induced stress waves," Laser-Tissue Interaction IV, S. L. Jacques and A. Katzir Ed., Vol. 1882, pp. 86-101, SPIE, Bellingham, 1993.

22. A. Vogel, W. Lauterborn, and R. Timm, "Optical and acoustic investigations of the dynamics of laser-produced cavitation bubbles near a solid boundary," J. Fluid Mech., Vol. 206, pp. 299-338, 1989.

23. B. Ward and D. C. Emmony, "Direct observation of the pressure developed in a liquid during cavitation-bubble collapse," Appl. Phys. Lett., Vol.59, pp.2228-2230, 1991.

24. A. G. Doukas, A. D. Zweig, J. K. Frisoli, R. Birngruber, and T. F. Deutsch, "Non-invasive determination of shock wave pressures generated by optical breakdown," Appl. Phys. B, Vol.53, pp. 237-245, 1991.

25. N. Do, K. Klees, A. C. Tam, P. T. Leung, and W. P. Leung, "Photodeflection probing of the explosion of a liquid film in contact with a solid heated by pulsed excimer laser irradiation," J. Appl. Phys., Vol. 74, pp. 1534-1538, 1993.

26. M. A. Harith, V. Palleschi, A. Salvetti, D. P. Singh, M. Vaselli, G. V. Dreiden, Y. I. Ostrovsky, and I. V. Semenova, "Dynamics of laser-driven shock waves in water," J. Appl. Phys. , Vol. 66, pp. 5194-5197, 1989.

27. X. Xu and C. P. Grigoropoulos, "High temperature radiative properties of thin silicon films at the $\lambda=0.6328$ $\mu \mathrm{m}$ wavelength," Int. J. Heat Mass Transfer, Vol. 36. pp. 4163-4172, 1994.

28. M. Born and E. Wolf, Principles of Optics, 6th Ed., Pergamon, Exeter, United Kingdom, pp. 51-70, pp. 611-633, 1986.

29. C. T. Avedisian, "The homogeneous nucleation limits of liquids," J. Phys. Chem. Ref. Data, Vol. 14, pp. $695-729,1985$. 